\title{
$-O$ \\ Computation of the Nucleolus for a Class of Disjunctive Games with a Permission Structure
}

René van den Brink ${ }^{7}$

Ilya Katsev²

Gerard van der Laan'

${ }^{\prime}$ Dept. of Econometrics, VU University Amsterdam, and Tinbergen Institute;

2 St. Petersburg Institute of Economics and Mathematics, Russian Academy of Sciences, St. Petersburg. 


\section{Tinbergen Institute}

The Tinbergen Institute is the institute for economic research of the Erasmus Universiteit Rotterdam, Universiteit van Amsterdam, and Vrije Universiteit Amsterdam.

Tinbergen Institute Amsterdam

Roetersstraat 31

1018 WB Amsterdam

The Netherlands

Tel.: +31(0)205513500

Fax: $+31(0) 205513555$

Tinbergen Institute Rotterdam

Burg. Oudlaan 50

3062 PA Rotterdam

The Netherlands

Tel.: + $31(0) 104088900$

Fax: $+31(0) 104089031$

Most TI discussion papers can be downloaded at http://www.tinbergen.nl. 


\title{
Computation of the nucleolus for a class of disjunctive games with a permission structure ${ }^{1}$
}

\author{
René van den Brink ${ }^{2}$ Ilya Katsev ${ }^{3}$ Gerard van der Laan ${ }^{4}$
}

June 9, 2008

\footnotetext{
${ }^{1}$ This research has been done while the second author was visiting the Tinbergen Institute, VU Amsterdam, on NWO-grant 047.017.017 within the framework of Dutch-Russian cooperation.

${ }^{2}$ J.R. van den Brink, Department of Econometrics and Tinbergen Institute, VU Amsterdam, De Boelelaan 1105, 1081 HV Amsterdam, The Netherlands. E-mail: jrbrink@feweb.vu.nl

${ }^{3}$ I.V. Katsev, St.Petersburg Institute for Economics and Mathematics, Russian Academy of Sciences, Tchaikovsky st.,1 191187 St.Petersburg, Russia. E-mail: katsev@yandex.ru

${ }^{4}$ G. van der Laan, Department of Econometrics and Tinbergen Institute, VU Amsterdam, De Boelelaan 1105, 1081 HV Amsterdam, The Netherlands. E-mail: glaan@feweb.vu.nl
} 


\begin{abstract}
A situation in which a finite set of players can obtain certain payoffs by cooperation can be described by a cooperative game with transferable utility, or simply a TU-game. A (single-valued) solution for TU-games assigns a payoff distribution to every TU-game. A well-known solution is the nucleolus. A cooperative game with a permission structure describes a situation in which players in a cooperative TU-game are hierarchically ordered in the sense that there are players that need permission from other players before they are allowed to cooperate. The corresponding restricted game takes account of the limited cooperation possibilities by assigning to every coalition the worth of its largest feasible subset. In this paper we provide a polynomial time algorithm for computing the nucleolus of the restricted games corresponding to a class of games with permission structure.
\end{abstract}

Keywords: TU-game, nucleolus, game with permission structure, peer group game, information market game, algorithm, complexity.

AMS subject classification: 91A12, 5C20

JEL code: C71 


\section{Introduction}

A cooperative game with transferable utility, or simply a TU-game is a finite set of players and for any subset (coalition) of players a worth representing the total payoff that the coalition can obtain by cooperating. A payoff vector is a vector which gives a payoff to each of the players, i.e., each component corresponds to precisely one of the players. A payoff vector is efficient if the sum of the payoffs is equal to the worth of the grand coalition of all players. A set-valued solution for TU-games assigns a set of payoff vectors (possibly empty) to every TU-game. A single-valued solution assigns precisely one payoff vector to every TU-game. A solution is said to be efficient if for every game any payoff vector assigned by the solution is efficient. The most well-known efficient set-valued solution is the Core (Gillies, 1953). The two most well-known efficient single-valued solutions are the Shapley value (Shapley, 1953) and the nucleolus (Schmeidler, 1969).

In this paper we assume that the players in a TU-game are part of some hierarchical structure that is represented by a directed graph such that some players need permission from other players before they are allowed to cooperate within a coalition. In the literature two approaches to these games with a permission structure can be found. In the conjunctive approach, as considered in Gilles et al. (1992) and van den Brink and Gilles (1996), it is assumed that each player needs permission from all its predecessors in the directed graph before it is allowed to cooperate. Alternatively, in the disjunctive approach as developed in Gilles and Owen (1994) and van den Brink (1997), a player needs permission to cooperate of at least one of its direct predecessors (if it has any). So, according to the conjunctive approach a coalition is feasible if and only if for any player in the coalition it holds that all its predecessors are also in the coalition, whereas according to the disjunctive approach a coalition is feasible if and only if for any player in the coalition at least one of its predecessors (if it has any) is also in the coalition. Following an approach similar to that of Myerson (1977) for games with limited communication (graph) structure, in both the conjunctive and disjunctive approach to games with a permission structure a restricted game is derived. In games with permission structure the restricted game assigns to every coalition the worth of its largest feasible subset. Applying well-known solutions as the Shapley value, Core or nucleolus to such restricted games yields solutions for games with a permission structure.

A special subclass of games with a permission structure arises from peer group situations, as introduced in Brânzei et al. (2002). A peer group situation is a triple consisting of a set of players, a hierarchical structure represented by a rooted directed tree, and for each player a real number representing his potential individual (economic) contribution to the society of all players. This yields an associated TU-game being the additive game in which the worth of any coalition is equal to the sum of the individual potentials of its 
members. In a rooted directed tree there is one top node (not having a predecessor), while any other node has precisely one predecessor. So, in case the hierarchical structure on the player set is a rooted directed tree, the conjunctive approach and the disjunctive approach as described above, coincide. The restricted game of the associated TU-game with respect to such a permission structure is called a peer group game. These peer group games have many interesting applications, see Brânzei et al. (2002). Clearly, in a peer group game the worth of a coalition is the sum of the individual potentials of the members of the largest feasible subset of the coalition. Since the top player is always in this set when he belongs to the coalition, and the largest feasible set is the empty set for any coalition not containing the unique top player, it follows that the top player is a veto player, i.e., any coalition not containing the (veto) top player has zero worth in the restricted game.

In Arin and Feltkamp (1997) an exponential time algorithm has been given to compute the nucleolus for veto-rich games. In Brânzei et al. (2005) a polynomial time algorithm is given to compute the nucleolus of a peer group game. In this paper we modify the Arin-Feltkamp algorithm to compute the nucleolus of the restricted game induced by more general situations, including peer group situations and information market situations (see Muto et al. (1989)) as special cases. The generalization concerns both the hierarchical graph structure and the class of unrestricted TU-games by allowing for any digraph having one top node and no directed cycles and any game satisfying a so-called weak digraph monotonicity condition and a weak digraph concavity condition. The algorithm finds the nucleolus in polynomial time.

The paper is organized as follows. Section 2 is a preliminary section containing cooperative TU-games (with special attention for the nucleolus), directed graphs and games with a permission structure. In section 3 we introduce the properties of weak digraph monotonicity and weak digraph concavity and we present some examples satisfying these conditions. In Section 4 we present some properties of essential and feasible coalitions. These properties are crucial for the algorithm given in Section 5. In Section 6 we discuss the complexity of the algorithm. Finally, Section 7 contains some concluding remarks.

\section{Preliminaries}

\section{$2.1 \quad$ TU-games}

A situation in which a finite set of players can obtain certain payoffs by cooperation can be described by a cooperative game with transferable utility, or simply a TU-game, being a pair $(N, v)$, where $N \subset \mathbb{N}$ is a finite set of $n$ players and $v: 2^{N} \rightarrow \mathbb{R}$ is a characteristic function on $N$ such that $v(\emptyset)=0$. For any coalition $S \subseteq N, v(S)$ is the worth of coalition $S$, i.e., the members of coalition $S$ can obtain a total payoff of $v(S)$ by agreeing to cooperate. For 
simplicity, for a single player $i$ we denote its worth $v(\{i\})$ by $v(i)$. We denote the collection of all characteristic functions on $N$ by $\mathcal{G}^{N}$. A TU-game $(N, v)$ is monotone if $v(S) \leq v(T)$ for all $S \subseteq T \subseteq N$. It is convex (concave) if $v(S)+v(T) \leq(\geq) v(S \cap T)+v(S \cup T)$ for all $S, T \subseteq N$.

A payoff vector is a vector $x \in \mathbb{R}^{n}$ assigning a payoff $x_{i}$ to every $i \in N$. In the sequel, for $S \subseteq N$ we denote $x(S)=\sum_{i \in S} x_{i}$. A payoff vector is efficient if $x(N)=v(N)$ and it is individually rational if $x_{i} \geq v(i)$ for every $i \in N$. The imputation set $I(N, v)$ of game $v$ is given by

$$
I(N, v)=\left\{x \in \mathbb{R}^{n} \mid x(N)=v(N) \text { and } x_{i} \geq v(i) \text { for every } i \in N\right\},
$$

i.e., $I(N, v)$ is the set of all efficient and individually rational payoff vectors. A (set-valued) solution $F$ on $\mathcal{G}^{N}$ assigns a set $F(N, v) \subset \mathbb{R}^{n}$ of payoff vectors to every characteristic function $v \in \mathcal{G}^{N}$. The most well-known set-valued solution is the Core assigning to every $v \in \mathcal{G}^{N}$ the set

$$
C(N, v)=\{x \in I(N, v) \mid x(S) \geq v(S) \text { for all } S \subset N\},
$$

i.e., it is the set of all imputations that are stable in the sense that no coalition can do better by separating from the grand coalition. The Core of $(N, v)$ is non-empty if and only if the game is balanced, see e.g. Bondareva (1962) or Shapley (1967). A collection $B=\left\{S_{1}, \ldots, S_{m}\right\}$ of subsets of $N$ is said to be a balanced collection when the system of equations

$$
\sum_{j=1}^{m} \lambda_{j} e^{S_{j}}=e^{N}
$$

has a unique positive solution, denoted by $\lambda_{j}^{B}, j=1, \ldots, m$, where, for $S \subseteq N$, the $n$-vector $e^{S}$ is given by $e_{i}^{S}=1$ when $i \in S$ and $e_{i}^{S}=0$ otherwise. A game $(N, v)$ is balanced if

$$
\sum_{j=1}^{m} \lambda_{j}^{B} v\left(S_{j}\right) \leq v(N)
$$

for any balanced collection $B=\left\{S_{1}, \ldots, S_{m}\right\}$.

Another (set-valued) solution is the Kernel assigning to every $v \in \mathcal{G}^{N}$ the set

$K(N, v)=\left\{x \in I(N, v) \mid\left[s_{i j}(x)=s_{j i}(x)\right]\right.$ or $\left[s_{i j}(x)>s_{j i}(x)\right.$ and $\left.x_{j}=v(j)\right]$ for all $\left.i, j \in N\right\}$, i.e., the set of all imputations such that for each pair of players $i$ and $j$ the complaint $s_{i j}(x)=\max \{v(S)-x(S) \mid i \in S, j \notin S\}$ of $i$ against $j$ is at least equal to the complaint of $j$ against $i$, with equality whenever $j$ gets more than its individual worth $v(j)$.

A solution $F$ is said to be single-valued if it assigns to any $v \in \mathcal{G}^{N}$ a unique payoff vector. The two most well-known single-valued solutions are the Shapley value (Shapley, 
1953) and the nucleolus (Schmeidler, 1969). Since the aim of this paper is to give an algorithm for computing the nucleolus for a special class of characteristic functions, we devote the next subsection to this solution.

\subsection{Nucleolus}

Consider a given a characteristic function $v \in \mathcal{G}^{N}$, and payoff vector $x \in \mathbb{R}^{n}$. Then the excess $e(S, x)$ of a coalition $S \subseteq N$ is defined by

$$
e(S, x)=v(S)-x(S)
$$

Further, let $E(x)$ be the $\left(2^{n}-2\right)$-component vector that is composed of the excesses of all coalitions $S \subset N, S \neq \emptyset$, in a non-increasing order, so $E_{1}(x) \geq E_{2}(x) \geq \ldots \geq$ $E_{2^{n}-2}(x)$. Then the nucleolus $N u c(N, v)$ of the game $(N, v)$ is the unique imputation which lexicographically minimizes the vector-valued function $E(\cdot)$ over the imputation set. Formally,

$$
N u c(N, v)=x \in I(N, v) \text { such that } E(x) \preceq_{L} E(y) \text { for all } y \in I(N, v),
$$

where $\preceq_{L}$ denotes the lexicographic order of vectors. It is well-known that $N u c(N, v) \in$ $K(N, v)$ and that $N u c(N, v) \in C(N, v)$ when $C(N, v) \neq \emptyset$. So, when the game has a nonempty Core, then the nucleolus is in the intersection of the Kernel and the Core.

In a game $(N, v)$, a coalition $S$ is called inessential if it has a partition $\left\{S_{1}, \ldots, S_{r}\right\}$ with $r \geq 2$, such that $v(S) \leq \sum_{j=1}^{r} v\left(S_{j}\right)$. Coalitions which are not inessential are called essential. Notice that single player coalitions are always essential. It is straightforward to observe that for an inessential coalition $S$ it holds that

$$
e(S, x) \leq \sum_{j=1}^{r} e\left(S_{j}, x\right), \text { for all } x \in \mathbb{R}^{n} .
$$

Therefore the Core, and thus also the nucleolus, is independent of inessential coalitions, as was noticed by Huberman (1980). In fact, in any $n$ player game there are at most $(2 n-2)$ coalitions which actually determine the nucleolus, see Brune (1983) and Reijnierse and Potters (1998). Although, as noticed by Brânzei et al. (2005), identifying these coalitions is no less laborious as computing the nucleolus itself, in the following we state some facts for games with non-empty Core which will appear to be useful later on. We denote

$$
e^{*}(N, v)=\min _{\{S \subset N \mid S \neq \emptyset\}}-e(S, x) \text { at } x=N u c(N, v),
$$

i.e., $e^{*}(N, v)$ the minimal negative excess at the nucleolus of game $(N, v)$. Clearly, $e^{*}(N, v) \geq$ 0 if and only if $\operatorname{Core}(N, v) \neq \emptyset$. 
Lemma 2.1 If $e^{*}(N, v)>0$, then every coalition $S \subset N$ with $-e(S, x)=e^{*}(N, v)$ at $x=N u c(N, v)$ is essential.

Proof. Suppose $S \subset N$ with $-e(S, x)=e^{*}(N, v)$ is inessential. Then there is a partition $\left\{S_{1}, \ldots, S_{m}\right\}$ such that $e^{*}(N, v)=-e(S, x) \geq \sum_{j=1}^{m}-e\left(S_{j}, x\right)$. Since $e^{*}(N, v)>0$ there must be at least one $j \in\{1, \ldots, m\}$ such that $-e\left(S_{j}, x\right)<-e(S, x)$, which contradicts that $e^{*}(N, v)=\min _{\{S \subset N \mid S \neq \emptyset\}}-e(S, x)$.

For the next lemma, let $B=\left\{S_{1}, \ldots, S_{m}\right\}$ be a balanced collection of coalitions and let $\mathcal{B}$ denote the set of all balanced collections, excluding the balanced collection $\{N\}$ having the grand coalition $N$ as its single element.

Lemma 2.2 If $e^{*}(N, v) \geq 0$ then

$$
e^{*}(N, v)=\min _{B \in \mathcal{B}} \frac{v(N)-\sum_{j=1}^{m} \lambda_{j}^{B} v\left(S_{j}\right)}{\sum_{j=1}^{m} \lambda_{j}^{B}},
$$

with $\lambda_{j}^{B}, j=1, \ldots, m$, the solution of the system (2.1) for the balanced collection $B=$ $\left\{S_{1}, \ldots, S_{m}\right\}$.

Proof. Let $B=\left\{S_{1}, \ldots, S_{m}\right\}$ be a balanced collection with $\lambda_{1}^{B}, . ., \lambda_{m}^{B}$ as the corresponding solution of system (2.1). Observe that for every $i \in N$ it holds that $\sum_{\left\{j \mid i \in S_{j}\right\}} \lambda_{j}^{B}=1$ and thus for every $x \in \mathbb{R}^{n}$ and $S \subset N$ we have that $x(S)=\sum_{i \in S} x_{i}=\sum_{i \in S} \sum_{\left\{j \mid i \in S_{j}\right\}} \lambda_{j}^{B} x_{i}$. Hence,

$$
\sum_{j=1}^{m} \lambda_{j}^{B} x\left(S_{j}\right)=\sum_{j=1}^{m} \sum_{i \in S_{j}} \lambda_{j}^{B} x_{i}=\sum_{i \in N} \sum_{\left\{j \mid i \in S_{j}\right\}} \lambda_{j}^{B} x_{i}=x(N)
$$

and thus at $x=N u c(N, v)$ we have that the convex combination $\sum_{j=1}^{m} \frac{\lambda_{j}^{B}}{\sum_{h} \lambda_{h}^{B}} \cdot\left(-e\left(S_{j}, x\right)\right)$ of all negative excesses $-e\left(S_{j}, x\right), j=1, \ldots, m$, is equal to

$$
\sum_{j=1}^{m} \frac{\lambda_{j}^{B}}{\sum_{h} \lambda_{h}^{B}} \cdot\left(x\left(S_{j}\right)-v\left(S_{j}\right)\right)=\frac{v(N)-\sum_{j=1}^{m} \lambda_{j}^{B} v\left(S_{j}\right)}{\sum_{h} \lambda_{h}^{B}} .
$$

Since every $-e\left(S_{j}, x\right) \geq e^{*}(N, v), j=1, \ldots, m$, also its convex combination is at least equal to $e^{*}(N, v)$, which shows that

$$
e^{*}(N, v) \leq \frac{v(N)-\sum_{j=1}^{m} \lambda_{j}^{B} v\left(S_{j}\right)}{\sum_{h} \lambda_{h}^{B}}
$$

Finally, from Kohlberg's theorem (see Kohlberg 1971) we know that there exists a balanced collection $B=\left\{S_{1}, \ldots, S_{m}\right\}$ with $e^{*}(N, v)=-e\left(S_{j}, x\right)$ for all $j$. For such a balanced collection inequality (2.2) holds with equality, which proves the lemma.

The next two corollaries follow immediately. 
Corollary 2.3 Let $B=\left\{S_{1}, \ldots, S_{m}\right\}$ be a balanced collection with weights $\lambda_{j}^{B}, j=$ $1, \ldots, m$, satisfying

$$
e^{*}(N, v)=\frac{v(N)-\sum_{j=1}^{m} \lambda_{j}^{B} v\left(S_{j}\right)}{\sum_{j=1}^{m} \lambda_{j}^{B}} .
$$

Then at $x=N u c(N, v)$ we have that $-e\left(S_{j}, x\right)=e^{*}(N, v), j=1, \ldots, m$.

Proof. As shown in the proof of Lemma 2.2, the right-hand side of equation (2.3) is a convex combination of the numbers $-e\left(S_{j}, x\right)$. Therefore, for each $j, e^{*}(N, v) \leq-e\left(S_{j}, x\right)$ must hold with equality.

Corollary 2.4 If $e^{*}(N, v)>0$, then for any balanced collection $B=\left\{S_{1}, \ldots, S_{m}\right\}$ satisfying $e^{*}(N, v)=\frac{v(N)-\sum_{j=1}^{m} \lambda_{j}^{B} v\left(S_{j}\right)}{\sum_{j=1}^{m} \lambda_{j}^{B}}$, it holds that any set $S_{j}$ is essential.

Proof. This follows immediately from Lemma 2.1 and Corollary 2.3.

Arin and Feltkamp (1997) propose an algorithm to find the nucleolus of a veto-rich game, i.e., a game $(N, v)$ such that there exists (at least one) veto player being a player $i$ such that $v(S)=0$ when $i \notin S$. The algorithm makes use of the fact that for veto-rich games the Kernel contains precisely one payoff vector, and thus the nucleolus is this unique element of the Kernel. For an element $x$ in the Kernel they first show that for any player $j$ it holds that $x_{j}=0$ if there exists $S \subseteq N \backslash\{j\}$ such that $v(S) \geq v(N)$. The algorithm is initiated by setting $x_{j}=0$ for all these players and setting $A_{0}$ as the set of these players. Observe that this set does not contain the set of veto players (unless it is the null-game and all players get zero payoff). It is also shown that the nucleolus payoff $x_{j}>0$ for all other players $j \in N \backslash A_{0}$ (thus including all veto players). After this initialisation the algorithm iteratively determines the payoffs of the other players as follows. Let $i$ be an arbitrarily chosen veto player. Then, at each step $t$, let $A_{t-1}$ be the set of players for which the payoffs have been determined already and let $B_{t}$ be the collection of all sets $S$ such that $i \in S$ and $\left(N \backslash A_{t-1}\right) \backslash S \neq \emptyset$. Then

$$
q_{t}=\min _{S \in B_{t}} q_{t}(S)
$$

where $q_{t}(S)=\frac{v(N)-v(S)-x\left(A_{t-1} \backslash S\right)}{\left|\left(N \backslash A_{t-1}\right) \backslash S\right|+1}$ is determined, and for any player $j \in \cap\left\{S \in B_{t} \mid q_{t}(S)=\right.$ $\left.q_{t}\right\}$ the nucleolus is set equal to $x_{j}=q_{t}$.

At any step $t$ the payoff of at least one player is determined, so in at most $n-1$ steps all payoffs $x_{j}, j \neq i$ are determined. As soon as all these payoffs are determined, the payoff $x_{i}$ of the chosen veto player $i$ is set equal to $v(N)-x(N \backslash\{i\})$. In the underlying paper we modify this algorithm to find the nucleolus of restricted games arising from games with a permission structure in which players in a cooperative TU-game belong to a hierarchical structure that is represented by a directed graph. 


\section{$2.3 \quad$ Directed graphs}

A directed graph or digraph is a pair $(N, D)$ where $N \subset \mathbb{N}$ is a finite set of nodes (representing the players) and $D \subseteq N \times N$ is a binary relation on $N$. Given $(N, D)$ and $S \subseteq N$, the digraph $(S, D(S))$ is the subgraph on $S$ given by $D(S)=\{(i, j) \in D \mid i, j \in S\}$. In the sequel we simply refer to $D$ for a digraph $(N, D)$ and to $D(S)$ for the subgraph $(S, D(S))$. For $i \in N$ the nodes in $S_{D}(i):=\{j \in N \mid(i, j) \in D\}$ are called the successors of $i$, and the nodes in $P_{D}(i):=\{j \in N \mid(j, i) \in D\}$ are called the predecessors of $i$.

For given $D$ on $N$, a path between $i$ and $j$ in $N$ is a sequence of distinct nodes $\left(i_{1}, \ldots, i_{m}\right)$ such that $i_{1}=i, i_{m}=j$, and $\left\{\left(i_{k}, i_{k+1}\right),\left(i_{k+1}, i_{k}\right)\right\} \cap D \neq \emptyset$ for $k=1, \ldots, m-1$. A set of nodes $T \subseteq N$ is connected in digraph $D$ if there is a path between any two nodes in $T$ that only uses arcs between nodes in $T$, i.e., if for every $i, j \in T$ there is a path $\left(i_{1}, \ldots, i_{m}\right)$ between $i$ and $j$ such that $\left\{i_{1}, \ldots, i_{m}\right\} \subseteq T$. A component in $D$ is a maximally connected set $T$ of nodes, i.e. $T$ is connected and $T \cup\{i\}$ is not connected for every $i \in N \backslash T$. A path $\left(i_{1}, \ldots, i_{m}\right)$ between $i$ and $j$ in $D$ is a directed path if $\left(i_{k}, i_{k+1}\right) \in D$ for $k=1, \ldots, m-1$. A directed path $\left(i_{1}, \ldots, i_{m}\right), m \geq 1$, in $D$ is a cycle if $\left(i_{m}, i_{1}\right) \in D$. We call digraph $D$ acyclic if it does not contain any cycle. Note that acyclicity of a digraph $D$ implies that $D$ is irrefexive, i.e., $(i, i) \notin D$ for all $i \in N$.

A digraph is called quasi-strongly connected if there exists a node $i_{0} \in N$, such that for every $j \neq i_{0}$ there is a directed path from $i_{0}$ to $j$. Note that this implies that $N$ is connected. When $D$ is acyclic then $i_{0}$ is the unique node in $N$ having no predecessors and $i_{0}$ is called the top-node of the digraph. The collection of all acyclic, quasi-strongly connected digraphs on $N$ is denoted by $\mathcal{D}^{N}$. A digraph $D \in \mathcal{D}^{N}$ is a rooted directed tree with root $i_{0}$ if there is precisely one path from the top-node $i_{0}$ to every other node. Node $j \in N$ is a complete subordinate of node $i \in N$ in $D \in \mathcal{D}^{N}$ if every directed path from the top-node $i_{0}$ to node $j$ contains node $i$. We denote the set of complete subordinates of node $i$ by $\bar{S}_{D}(i)$, i.e.,

$$
\bar{S}_{D}(i)=\left\{j \in N \mid \begin{array}{c}
i \in\left\{h_{1}, \ldots, h_{t-1}\right\} \text { for every sequence of nodes } h_{1}, \ldots, h_{t} \\
\text { such that } h_{1}=i_{0}, h_{k+1} \in S_{D}\left(h_{k}\right), k \in\{1, \ldots, t-1\}, \text { and } h_{t}=j
\end{array}\right\} .
$$

\subsection{Restricted games}

In this paper we assume that the players in a TU-game are part of a hierarchical structure that is represented by a directed graph, refered to as a permission structure, such that some players need permission from other players before they are allowed to cooperate within a coalition. A triple $(N, v, D)$ with $(N, v)$ a TU-game and $(N, D)$ a digraph with the player set $N$ as the set of nodes is called a game with a permission structure. In the sequel we 
assume that $D \in \mathcal{D}^{N}$ and (without loss of generality) that $i_{0}=1$ is its unique top-node ${ }^{1}$.

Assumption $2.5(N, D)$ is acyclic and quasi-strongly connected with $P_{D}(1)=\emptyset$ (and thus $P_{D}(i) \neq \emptyset$ for every $\left.i \neq 1\right)$.

As noticed in the introduction we can distinguish between the conjunctive and disjunctive approach. In this paper we consider the disjunctive approach as developed in Gilles and Owen (1994) and van den Brink (1997), where a player $i \neq 1$ needs permission to cooperate of at least one of its direct predecessors. Therefore a coalition is feasible if and only if it contains the top-player 1 and for every other player in the coalition at least one of its predecessors is also in the coalition. So, for digraph $(N, D)$, the set of disjunctive feasible coalitions is given by

$$
\Phi^{D}=\left\{S \subseteq N \mid P_{D}(i) \cap S \neq \emptyset \text { for all } i \in S \backslash\{1\}\right\}
$$

For any $S \subseteq N$, let $\sigma(S)=\cup\left\{T \in \Phi^{D} \mid T \subseteq S\right\}$ be the largest disjunctive feasible subset of $S$ in $D .^{2}$ By Assumption 2.5 we have that for every $i \neq 1$, there is at least one directed path from 1 to $i$. As a consequence it follows that for every $S \subseteq N$ with $\sigma(S) \neq \emptyset$, the subgraph $(\sigma(S), D(\sigma(S))$ is acyclic and quasi-strongly connected with node $1 \in \sigma(S)$ as its unique top-node.

Given the triple $(N, v, D)$ with $v \in \mathcal{G}^{N}$ and $D \in \mathcal{D}^{N}$, under the disjunctive permission structure the induced restricted game $r: 2^{N} \rightarrow \mathbb{R}$ is given by

$$
r(S)=v(\sigma(S)) \text { for all } S \subseteq N .
$$

Since player 1 is the top-node it holds that $r(S)=0$ when $1 \notin S$, i.e., the restricted game is a veto-rich game with respect to the top-player 1 . If $D$ is a rooted directed tree (with node 1 as its root), then $\left|P_{D}(i)\right|=1$ for all $i \neq 1$ and the conjunctive and disjunctive approach coincide. In this case the triple $(N, v, D)$ is a peer group situation when the game $(N, v)$ is a non-negative additive game (see Brânzei et. al. (2002)), i.e., there exist real numbers $a_{i}, i \in N$, such that $v(S)=\sum_{i \in S} a_{i}, S \subseteq N$. Then the restricted game $(N, r)$ as defined in (2.5) is a so-called peer group game and is given by

$$
r(S)=v(\sigma(S))=\sum_{\left\{i \in S \mid \widehat{P}_{D}(i) \subseteq S\right\}} a_{i}
$$

where $j \in \widehat{P}_{D}(i)$ if and only if if there exists a sequence of players $\left(h_{1}, \ldots, h_{t}\right)$ such that $h_{1}=j, h_{k+1} \in S\left(h_{k}\right)$ for all $1 \leq k \leq t-1$, and $h_{t}=i$. A peer group game $(N, r)$ is a

\footnotetext{
${ }^{1}$ This implies that $1 \in N$. Later we consider reduced games on proper subsets of $N^{\prime} \subset N$, but the top-player 1 always belongs to $N^{\prime}$.

${ }^{2}$ Every coalition having a unique largest feasible subset follows from $\Phi^{D}$ being closed under union.
} 
monotone veto-rich game and has a non-empty Core. In particular (with 1 the veto player) the payoff vector $x \in \mathbb{R}_{+}^{n}$ given by $x_{1}=v(N)$ and $x_{i}=0, i \neq 1$, belongs to the Core. As noticed in Brânzei et al. (2002), this class of games with permission structure contains several interesting applications.

\section{Weak digraph monotonicity and concavity}

The algorithm to be presented in Section 5 holds for games with a permission structure $(N, v, D)$ satisfying the next two conditions. First, we say that a game with permission structure $(N, v, D)$ satisfies weak digraph monotonicity if

$$
\left[S \subseteq N \text { and } S \in \Phi^{D}\right] \Rightarrow v(S) \leq v(N) .
$$

Observe that weak digraph monotonicity weakens monotonicity in two respects, namely (i) the monotocity condition $v(S) \leq v(T)$ if $S \subseteq T$ only has to hold for $T=N$ and (ii) for sets $S$ that are feasible given the disjunctive permission structure on the digraph $D$. Second, we say that a game with permission structure $(N, v, D)$ satisfies weak digraph concavity if

$$
\left[S \cup T=N \text { and } S, T \in \Phi^{D}\right] \Rightarrow v(S)+v(T) \geq v(S \cap T)+v(N) .
$$

Observe that also this property weakens the concavity of a game in two respects, namely that the concavity condition only has to hold for sets that $S$ and $T$ satisfying (i) $S \cup T=N$ and (ii) $S$ and $T$ are feasible given the disjunctive permission structure on $D$. So, for both properties the adjunctive 'weak' means that the inequality conditions are only required for $T=N$, respectively $S \cup T=N$, and the adjunctive 'digraph' means that the inequality conditions are only required for feasible sets with respect to the permission structure. Monotonicity is a condition satisfied by most of the games that arise from economic or social situations, so this is certainly the case for weak digraph monotonicity. Although concavity is a strong condition for profit games ${ }^{3}$, weak digraph concavity is considerably weaker and is also satisfied by several interesting classes of profit games with permission structure. We give some examples.

\section{Example 3.1 Generalised peer group situations}

It is obvious that peer group situations $(N, v, D)$ as mentioned at the end of Subsection 2.4 satisfy weak digraph monotonicity. Further, for any feasible $S$ and $T$ such that $S \cup T=N$ we have that $S \cap T$ is feasible (since $D$ is a rooted tree) and

$$
v(S)+v(T)=\sum_{i \in S} a_{i}+\sum_{i \in T} a_{i}=\sum_{i \in S \cap T} a_{i}+\sum_{i \in N} a_{i}=v(S \cap T)+v(N) .
$$

\footnotetext{
${ }^{3}$ Given our nucleolus concept in which the maximum excess $v(S)-x(S)$ is minimized, in this paper we deal with profit games.
} 
So, $(N, v, D)$ also satisfies weak digraph concavity.

We say that $(N, v, D)$ is a generalised peer group situation when $D \in \mathcal{D}^{N}$ is an acyclic and quasi-strongly connected digraph (and $v$ is again a nonnegative additive game). Clearly, any generalised peer group situation satisfies weak digraph monotonicity and weak digraph concavity. It now might happen that $S \cap T$ is not feasible under the disjunctive approach. Then the weak digraph concavity condition might hold with strict inequality.

\section{Example 3.2 Generalised information market situation}

Let $\mathcal{S}=\left\{S_{1}, \ldots, S_{K}\right\}$ be a collection of $K$ (nonempty) subsets of $N$, and $\alpha_{k}, k=1, \ldots, K$, be positive numbers. Then we consider the game $(N, v)$ given by

$$
v(S)=\sum_{\left\{k \mid S_{k} \cap S \neq \emptyset\right\}} \alpha_{k}, \quad S \subseteq N .
$$

Further, let $D \in \mathcal{D}^{N}$ be any digraph satisfying $(1, j) \in D$ for all $j \in\{2, \ldots, n\}$. So, $j=1$ is the top-player and $S \subseteq N$ is feasible if and only if $1 \in S$. Now, the restricted game $(N, r)$ is given by $r(S)=0$ if $1 \notin S$ and

$$
r(S)=\sum_{\left\{k \mid S_{k} \cap S \neq \emptyset\right\}} \alpha_{k}, \text { if } 1 \in S .
$$

The game $(N, r)$ is an information game as introduced in Muto et al. (1989). Obviously, $(N, v, D)$ satisfies weak digraph monotonicity. Further, for any feasible $S$ and $T$ such that $S \cup T=N$ we have that $S \cap T$ is feasible and

$$
\begin{gathered}
v(S)+v(T)=\sum_{\left\{k \mid S_{k} \cap S \neq \emptyset\right\}} \alpha_{k}+\sum_{\left\{k \mid S_{k} \cap T \neq \emptyset\right\}} \alpha_{k}= \\
\sum_{\left\{k \mid S_{k} \cap(S \cap T) \neq \emptyset\right\}} \alpha_{k}+\sum_{\left\{k \mid S_{k} \cap N \neq \emptyset\right\}} \alpha_{k}=v(S \cap T)+v(N)
\end{gathered}
$$

where the last but one equality follows since $S \cup T=N$. Thus $(N, v, D)$ also satisfies weak digraph concavity. In fact, this condition is satisfied for any $D \in \mathcal{D}^{N}$. In case $S \cap T$ is not feasible the condition might hold with strict inequality.

Observe that also any game with permission structure $(N, v, D)$ where $v$ is the sum of an additive game and a game as given above in equation (3.8), satisfies the conditions of weak digraph monotonicity and weak digraph concavity.

\section{Example 3.3 Market situation}

Let the set $N$ consist of one seller, say player 1 , having one item for sale, and $n-1$ buyers, 
and let $a_{j}$ be the nonnegative surplus of trade between the seller and buyer $j, j=2, \ldots, n$. Then the market game is given by $(N, v)$ with $v(S)=0$ if $1 \notin S$ and

$$
v(S)=\max _{j \in S \backslash\{1\}} a_{j}, \text { if } 1 \in S .
$$

Further, let $D \in \mathcal{D}^{N}$ be any digraph satisfying $(1, j) \in D$ for all $j \in\{2, \ldots, n\}$. So, $S \subseteq N$ is feasible if and only if $1 \in S$. Then for any feasible $S$ and $T$ such that $S \cup T=N$ we have that

$$
v(N)=\max [v(S), v(T)]
$$

and

$$
v(S \cap T) \leq \min [v(S), v(T)]
$$

Hence

$$
v(S)+v(T)=\max [v(S), v(T)]+\min [v(S), v(T)] \geq v(N)+v(S \cap T) .
$$

Hence $(N, v, D)$ also satisfies weak digraph concavity. Clearly, it also satisfies weak digraph monotonicity.

\section{Essential and feasible coalitions}

In this section we prove several results of essential and feasible coalitions for games with permission structure $(N, v, D)$ that will be used later on to prove that the algorithm of Section 5 will indeed find the nucleolus of the restricted game. The first lemma does not yet require the two conditions (3.6) and (3.7) and says that any essential coalition with at least two elements is feasible.

Lemma 4.1 If $S \subseteq N$ with $|S| \geq 2$ is essential in the restricted game $(N, r)$, then $S$ is feasible.

Proof. Suppose that $S$ is not feasible. Then $r(S)=r(\sigma(S))$ with $\sigma(S) \subset S$. Since $r(j)=0$ for all $j \in S \backslash \sigma(S)$, it holds that $r(S)=r(\sigma(S))+\sum_{j \in S \backslash \sigma(S)} r(j)$, implying that $S$ is not essential.

Assume that $(N, v, D)$ satisfies condition (3.6). Then it follows that the restricted game $(N, r)$ is a weak monotone $(r(S) \leq r(N)$ for all $S \subseteq N)$ veto-rich game (with veto player 1) and therefore the Core contains the payoff vector $(r(N), 0, \ldots, 0)^{\top} \in \mathbb{R}^{n}$ and thus is not empty. (Observe that $r(N)=v(N)$.) Hence, $N u c(N, r)$ is in the Core of $(N, r)$ and independent of inessential coalitions. From now on we make the following assumption. 
Assumption $4.2 N$ is essential in the restricted game $(N, r)$.

In fact, when $(N, v, D)$ is weak digraph monotone, this assumption is without loss of generality. If $N$ is inessential then there exists a partition $\left\{S_{1}, \ldots, S_{m}\right\}$ such that (i) $r(N) \leq \sum_{j=1}^{m} r\left(S_{j}\right)$, (ii) $S_{1}$ is essential, and (iii) $1 \in S_{1}$. Because of (iii) we have that $S_{2}, \ldots, S_{m}$ are not feasible and thus $r\left(S_{j}\right)=0$ for $j=2, \ldots, m$. Together with weak digraph monotonicity this implies that $r(N)=r\left(S_{1}\right)$. So, according to Arin and Feltkamp (1997), the nucleolus assigns a zero payoff to every player not in $S_{1}$, and we can restrict ourselves to the subgame and subgraph on the essential coalition $S_{1}$ containing player 1 . For $N$ essential, also observe that according to Arin and Feltkamp (1997) the nucleolus assigns positive payoff to any player in $N$. Since the assumption that $N$ is essential in the game $(N, r)$ implies that $r(N)>r(S)$ for every $S \subset N$, we have the following lemma.

Lemma 4.3 If game with permission structure $(N, v, D)$ satisfies condition (3.6), then $e^{*}(N, r)>0$.

Proof. Since $C(N, r) \neq \emptyset$ we have that $e^{*}(N, r) \geq 0$. Hence, according to Lemma 2.2 it holds that

$$
e^{*}(N, r)=\min _{B \in \mathcal{B}} \frac{r(N)-\sum_{j=1}^{m} \lambda_{j}^{B} r\left(S_{j}\right)}{\sum_{j=1}^{m} \lambda_{j}^{B}},
$$

with $\lambda_{j}^{B}, j=1, \ldots, m$, the solution of the system (2.1) for the balanced collection $B$. Since $r\left(S_{j}\right)=0$ when $1 \notin S_{j}$, we obtain that

$$
e^{*}(N, r)=\min _{B \in \mathcal{B}} \frac{r(N)-\sum_{\left\{j \mid 1 \in S_{j}\right\}} \lambda_{j}^{B} r\left(S_{j}\right)}{\sum_{j=1}^{m} \lambda_{j}^{B}} .
$$

Since the collection $\{N\}$ does not belong to $\mathcal{B}$, any $S_{j}$ in a balanced collection $B$ is a real subset of $N$ and thus $r\left(S_{j}\right)<r(N)$ for any $S_{j}$, because $N$ is essential. Since $\sum_{\left\{j \mid 1 \in S_{j}\right\}} \lambda_{j}^{B}=$ 1 by the definition of balancedness, it follows that $r(N)-\sum_{\left\{j \mid 1 \in S_{j}\right\}} \lambda_{j}^{B} r\left(S_{j}\right)>0$ for any $B \in \mathcal{B}$, which proves the lemma.

Similar as in Arin and Feltkamp (1997), in the sequel we denote for $S \subset N$ and the restricted game $(N, r)$,

$$
\tau(S, r)=\frac{r(N)-r(S)}{|N \backslash S|+1} .
$$

In the following, $\Omega^{D}=\Phi^{D} \backslash\{N\}$ denotes the collection of all feasible coalitions not equal to $N$. We now have the following lemmas.

Lemma 4.4 Let game with permission structure $(N, v, D)$ satisfy condition (3.6). Then

$$
e^{*}(N, r)=\min _{S \in \Omega^{D}} \tau(S, r) .
$$


Proof. According to Kohlberg's theorem there exists a balanced collection $\left\{S_{1}, \ldots, S_{m}\right\}$ such that $-e\left(S_{k}, x\right)=e^{*}(N, r)$ for all $k=1, \ldots, m$. Since $e^{*}(N, r)>0$ by Lemma 4.3, according to Corollary 2.4 we have that any $S_{j}$ is essential. Without loss of generality, let $1 \in S_{1}$. Then, we have that either $S_{1}=\{1\}$ and thus feasible, or $\left|S_{1}\right|>1$ and thus feasible according to Lemma 4.1. Denote $U=S_{1}$. Now, consider $j \notin U$. Since the collection is balanced, there must be a coalition $S_{k} \neq S_{1}=U$ containing $j$, but not 1 . Then $S_{k}$ is essential, but not feasible. Hence it follows with Lemma 4.1 that $\left|S_{k}\right|=1$ and thus $S_{k}=\{j\}$. Now, let $\lambda_{U}^{B}$ and $\lambda_{j}^{B}, j \notin U$, be the corresponding weights. Then $\lambda_{U}^{B}=\lambda_{j}^{B}=1$, $j \notin U$. Further $r(j)=0$ for all $j \notin U$ since $\{j\}$ is not feasible. Substituting these values in (2.3) gives $e^{*}(N, r)=\frac{r(N)-\lambda_{U}^{B} r(U)}{|N \backslash U|+1}=\tau(U, r)$, showing that there exists a coalition $U \in \Omega^{D}$ satisfying $e^{*}(N, r)=\tau(U, r)$. Next, consider any $S \in \Omega^{D}$. Then $B=\{S\} \cup\{\{j\} \mid j \notin S\}$ is a balanced collection with corresponding weights $\lambda_{S}^{B}=\lambda_{j}^{B}=1, j \notin S$. Since $1 \in S$ (because $S$ is feasible), it follows that $r(j)=0$ for all $j \notin S$. Hence with Lemma 2.2 we obtain that that $e^{*}(N, r) \leq \frac{r(N)-\lambda_{S}^{B} r(S)-\sum_{j \notin S} \lambda_{j}^{B} r(j)}{|N \backslash S|+1}=\frac{r(N)-r(S)}{|N \backslash S|+1}=\tau(S, r)$.

Lemma 4.5 Let game with permission structure $(N, v, D)$ satisfy condition (3.6), let $U \in$ $\Omega^{D}$ be such that $\tau(U, r)=e^{*}(N, r)$, and let $y \in \mathbb{R}^{n}$ be such that $y(U)=r(U)+\tau(U, r)$ and $y_{j}=\tau(U, r)$ for all $j \notin U$. Then $x=N u c(N, r)$ satisfies $x(U)=y(U)$ and $x_{j}=y_{j}$ for all $j \notin U$.

Proof. First, observe that

$$
y(N)=y(U)+\sum_{j \notin U} y_{j}=r(U)+(|N \backslash U|+1) \tau(U, r)=r(N),
$$

so $y$ is efficient. Next, observe that $U$ is feasible and thus $1 \in U$. Hence for any $j \notin U$, the singleton coalition $\{j\}$ is not feasible and thus $r(j)=0$. Therefore the excesses for the coalitions $U \in \Phi^{D}$ and the singletons $\{j\}, j \notin U$, at $y$ are equal to $e(U, y)=-\tau(U, r)=$ $e(\{j\}, y), j \notin U$. Now, suppose that $x=N u c(N, r)$ does not satisfy $x(U)=y(U)$ and $x_{j}=y_{j}$. Then

$$
\min \left[-e(U, x), \min _{j \notin U}-e(\{j\}, x)\right]<\tau(U, r),
$$

contradicting that $\tau(U, r)=e^{*}(N, r)=\min _{\{S \subset N, S \neq \emptyset\}}-e(S, x)$.

The two lemmas above show that as soon as a coalition $U \in \Omega^{D}$ has been found with $\tau(U, r)=\min _{S \in \Omega^{D}} \tau(S, r)$, the nucleolus values of all players $j \notin U$ have been found and that these values are equal to $\tau(U, r)$. This gives us the basic idea for the algorithm 
in the next section. In the sequel, denote $\tau^{*}(r)=\min _{S \in \Omega^{D}} \tau(S, r)$. In the first step the algorithm searches for a coalition $U_{1} \in \Omega^{D}$ satisfying

$$
\tau\left(U_{1}, r\right)=\tau^{*}(r) \text { and }\left|U_{1}\right|=\max _{\left\{U \in \Omega^{D} \mid \tau(U, r)=\tau^{*}(r)\right\}}|U|,
$$

i.e., any other feasible set $U \neq N$ satisfying $\tau(U, r)=\tau^{*}(r)$ contains at most the same number of players as $U_{1}$. This gives nucleolus payoffs $\tau^{*}(r)=\tau\left(U_{1}, r\right)$ to any player $j \notin U_{1}$ and in the next step the algorithm continues with a search on a reduced set of players $U_{1}$. The details of the algortihm will be given in the next section. In the remaining of this section we give several results with respect to a set $U_{1}$ satisfying condition (4.9). These results will be used in Section 5 to prove that the algorithm indeed finds the nucleolus. Observe that the results above only require weak digraph monotonicity. The next results require both weak digraph monotonicity and weak digraph concavity.

Lemma 4.6 Let game with permission structure $(N, v, D)$ satisfy conditions (3.6) and (3.7) and, for a coalition $U_{1}$ satisfying condition (4.9), let $\left\{T_{1}, T_{2}\right\}$ be a partition of $N \backslash U_{1}$. Then at least one of the two coalitions $U_{1} \cup T_{1}, U_{1} \cup T_{2}$ is not feasible.

Proof. Suppose that both sets $U_{1} \cup T_{1}$ and $U_{1} \cup T_{2}$ are feasible. Then we have that

$$
\begin{gathered}
\frac{\left|T_{2}\right|+1}{\left|T_{1}\right|+\left|T_{2}\right|+2} \tau\left(U_{1} \cup T_{1}, r\right)+\frac{\left|T_{1}\right|+1}{\left|T_{1}\right|+\left|T_{2}\right|+2} \tau\left(U_{1} \cup T_{2}, r\right)= \\
=\frac{r(N)-r\left(N \backslash T_{2}\right)}{\left|T_{1}\right|+\left|T_{2}\right|+2}+\frac{r(N)-r\left(N \backslash T_{1}\right)}{\left|T_{1}\right|+\left|T_{2}\right|+2}= \\
=\frac{2 r(N)-r\left(N \backslash T_{1}\right)-r\left(N \backslash T_{2}\right)}{\left|T_{1}\right|+\left|T_{2}\right|+2} \leq \frac{r(N)-r\left(U_{1}\right)}{\left|T_{1}\right|+\left|T_{2}\right|+2},
\end{gathered}
$$

where the last inequality follows from condition (3.7) for the sets $N \backslash T_{j}, j=1,2$, since $r(N)=v(N), r\left(U_{1}\right)=v\left(U_{1}\right)$ by the feasibility of $U_{1}$, and for $i \in\{1,2\}, i \neq j$, we have that $r\left(N \backslash T_{j}\right)=r\left(U_{1} \cup T_{i}\right)=v\left(U_{1} \cup T_{i}\right)$ because of the feasibility of $U_{1} \cup T_{i}$. Further since $r\left(U_{1}\right)=v\left(U_{1}\right) \leq v(N)=r(N)$ because of condition (3.6), we have that

$$
\frac{r(N)-r\left(U_{1}\right)}{\left|T_{1}\right|+\left|T_{2}\right|+2} \leq \frac{r(N)-r\left(U_{1}\right)}{\left|T_{1}\right|+\left|T_{2}\right|+1}=\tau\left(U_{1}, r\right) .
$$

So, $\tau\left(U_{1}, r\right)$ is at least equal to the given convex combination of $\tau\left(U_{1} \cup T_{1}, r\right)$ and $\tau\left(U_{1} \cup\right.$ $\left.T_{2}, r\right)$, implying that for at least one $i, i=1,2$, it holds that

$$
\tau\left(U_{1} \cup T_{i}, r\right) \leq \tau\left(U_{1}, r\right) .
$$

This contradicts condition (4.9). 
The next proposition says that for a set $U_{1}$ satisfying condition (4.9) the complement $N \backslash U_{1}$ is connected and that the collection of all successors of players in $U_{1}$ contains precisely one player not in $U_{1}$. For $T \subseteq N$, let $S_{D}(T)=\cup_{i \in T} S_{D}(i)$ denote the union of all successors of at least one player of $T$ in the digraph $(N, D)$.

Proposition 4.7 Let game with permission structure $(N, v, D)$ satisfy conditions (3.6) and (3.7) and let $U_{1}$ be a coalition satisfying condition (4.9). Then:
1. The set $N \backslash U_{1}$ is connected,
2. $\left|S_{D}\left(U_{1}\right) \cap\left(N \backslash U_{1}\right)\right|=1$.

Proof. 1. To prove 1, suppose $N \backslash U_{1}$ consists of at least two components. Let $T_{1}$ be one of the components and denote $T_{2}=N \backslash\left(U_{1} \cup T_{1}\right)$. We show that both $U_{1} \cup T_{i}, i=1,2$ are feasible.

To do so, let $i$ be any player in $T_{1}$. By quasi-strongly connectedness of $(N, D)$, there exists a directed path $\left(i_{1}, i_{2}, \ldots, i_{m}\right)$ from $i_{1}=1$ to $i_{m}=i$. Let $i_{k}, 1 \leq k<m$, be the last player in the path not in $T_{1}$, thus $i_{k} \in U_{1} \cup T_{2}$ and $i_{k+1}, \ldots, i_{m} \in T_{1}$. Since $\left(i_{k}, i_{k+1}\right) \in D, i_{k} \in T_{2}$ contradicts that $T_{1}$ is a component of $N \backslash U_{1}$. Hence $i_{k} \in U_{1}$. Since $U_{1}$ is feasible, $1 \in U_{1}$ and there is a path $\left(j_{1}, \ldots, j_{\ell}\right)$ from $j_{1}=1$ to $j_{\ell}=i_{k}$ with $j_{r} \in U_{1}$ for all $r=1, \ldots, \ell$. Hence for any $i \in T_{1}$ there is a path $\left(j_{1}, \ldots, j_{\ell}, i_{k+1}, \ldots, i_{m}\right)$ from 1 to $i$ only containing nodes in $U_{1} \cup T_{1}$. This shows that $U_{1} \cup T_{1}$ is feasible. Similarly it follows that $U_{1} \cup T_{2}$ is feasible. This contradicts Lemma 4.6, which proves the first statement.

2. To prove 2 , assume that there are two players $i_{1}, i_{2} \in S_{D}\left(U_{1}\right) \cap\left(N \backslash U_{1}\right), i_{1} \neq i_{2}$. For any player $i \in N \backslash U_{1}$, let $\widetilde{S}_{D}(i)$ be defined as the subset of $N \backslash U_{1}$ such that node $j \in N \backslash U_{1}$ belongs to $\widetilde{S}_{D}(i)$ if and only if $j=i$ or there is a directed path from node $i$ to node $j$ that only consists of nodes in $N \backslash U_{1}$. Since $(N, D)$ is acyclic by assumption, we have that $i_{1} \notin \widetilde{S}_{D}\left(i_{2}\right)$ or $i_{2} \notin \widetilde{S}_{D}\left(i_{1}\right)$ (or both). Suppose $i_{2} \notin \widetilde{S}_{D}\left(i_{1}\right)$. We now consider the partition of $N \backslash U_{1}$ into two non-empty sets $T_{1}=\widetilde{S}_{D}\left(i_{1}\right)$ and $T_{2}=\left(N \backslash U_{1}\right) \backslash T_{1}$ and obtain a contradiction by using Lemma 4.6. Since there is a directed path from node 1 to $i_{1} \in T_{1}$ consisting of nodes in $U_{1} \cup\left\{i_{1}\right\}$, and from $i_{1} \in T_{1}$ to any other node in $T_{1}$ consisting of nodes in $T_{1}$, for each $j \in U_{1} \cup T_{1}$ there is a path in $U_{1} \cup T_{1}$ from 1 to $j$, and thus $U_{1} \cup T_{1}$ is feasible.

Next consider $U_{1} \cup T_{2}$. For a node $j \in T_{2}$, let $\left(i_{1}, i_{2}, \ldots, i_{m}\right)$ be a path from $i_{1}=1$ to $i_{m}=j$ and let $i_{k}, 1 \leq k<m$, be the last player in the path not in $T_{2}$, thus $i_{k} \in N \backslash T_{2}=U_{1} \cup T_{1}$. Then $i_{k} \in U_{1}$, because $i_{k} \in T_{1}=\widetilde{S}_{D}\left(i_{1}\right)$ contradicts that $j \notin T_{1}$. Since $U_{1}$ is feasible, there is a path $\left(j_{1}, \ldots, j_{\ell}\right)$ from $j_{1}=1$ to $j_{\ell}=i_{k}$ with $j_{r} \in U_{1}$ for all $r=1, \ldots, \ell$. Hence for any $j \in T_{2}$ there is a path $\left(j_{1}, \ldots, j_{\ell}, i_{k+1}, \ldots, i_{m}\right)$ from 1 to $j$ only containing nodes in $U_{1} \cup T_{2}$. This shows that $U_{1} \cup T_{2}$ is feasible. Hence the existence of two 
players in $S_{D}\left(U_{1}\right) \cap\left(N \backslash U_{1}\right)$ contradicts Lemma 4.6, which proves the second statement.

For $U_{1}$ satisfying condition (4.9), let $i_{1}$ be the unique node in $S_{D}\left(U_{1}\right) \cap\left(N \backslash U_{1}\right)$ i.e., $i_{1}$ is the unique successor of $U_{1}$ in $N \backslash U_{1}$. Since $1 \in U_{1}$, this implies that any path from node 1 to a player $j \in N \backslash U_{1}$ has node $i_{1}$ as the first player on the path not in $U_{1}$. Together with the connectedness of $N \backslash U_{1}$ (see Proposition 4.7) this gives the following corollary.

Corollary 4.8 Let game with permission structure ( $N, v, D)$ satisfy conditions (3.6) and (3.7) and let $U_{1}$ be a coalition satisfying condition (4.9). Then the subgraph $\left(N \backslash U_{1}, D(N \backslash\right.$ $\left.U_{1}\right)$ ) of $(N, D)$ on $N \backslash U_{1}$ is also a quasi-strongly connected, acyclic directed graph with one top-node (node $i_{1}$ ).

\section{An algorithm for computing the nucleolus}

Since disjunctive restricted games are veto-rich games we can apply the exponential time algorithm of Arin and Feltkamp (1997) to find the nucleolus of the restricted game $(N, r)$ of a game with permission structure $(N, v, D)$ that satisfies conditions (3.6) and (3.7). However, instead of directly applying the algorithm of Arin and Feltkamp to $(N, r)$, in this section we give a modified version of the algorithm which finds the nucleolus in polynomial time by making use of the hierarchical structure given by the digraph $(N, D)$. In particular the hierarchical structure reduces at each step $t$ the minimization problem to find $q_{t}$ (see (2.4)), because it is sufficient to consider the feasible sets.

Let node 1 be the unique top node in $(N, D)$, and thus 1 is a veto player in the restricted game $(N, r)$. We again assume that $(N, v, D)$ satisfies the conditions (3.6) and (3.7) and that $N$ is essential in $(N, r)$. Further for the reduced game with permission structure $\left(U_{k}, v_{k}, D_{k}\right)$ defined in iteration $k-1$ at Step 3 of the algorithm given below, the set $\Omega^{D_{k}}$ denotes the set of all feasible coalitions not equal to $U_{k}$ in the digraph $\left(U_{k}, D_{k}\right)$. Also, for $i \in U_{k}$, we denote by $S_{D_{k}}(i)$ and $P_{D_{k}}(i)$ the set of successors, respectively predecessors in $\left(U_{k}, D_{k}\right)$. Then the algorithm proceeds as follows.

\section{Algorithm}

Step 1 Set $k=0, U_{0}=N, v_{0}=v, D_{0}=D$ and $r_{0}=r$. Goto Step 2 .

Step 2 Find $U_{k+1} \subset U_{k}$ satisfying condition (4.9) with respect to game with permission structure $\left(U_{k}, v_{k}, D_{k}\right)$, i.e.,

$$
\tau\left(U_{k+1}, r_{k}\right)=\tau^{*}\left(r_{k}\right) \text { and }\left|U_{k+1}\right|=\max _{\left\{U \in \Omega^{D_{k}} \mid \tau\left(U, r_{k}\right)=\tau^{*}\left(r_{k}\right)\right\}}|U|,
$$


where $\tau^{*}\left(r_{k}\right)=\min _{U \in \Omega^{D_{k}}} \tau\left(U, r_{k}\right)$ with $\tau\left(U, r_{k}\right)=\frac{r_{k}\left(U_{k}\right)-r_{k}(U)}{\left|U_{k} \backslash U\right|+1}$. Assign $y_{j}=\tau^{*}\left(r_{k}\right)$ to every player $j \in U_{k} \backslash U_{k+1}$. Goto Step 3 .

Step 3 If $U_{k+1}=\{1\}$ then Goto Step 4. If $U_{k+1} \neq\{1\}$, let $i_{k+1}$ be the unique top-player of the subgraph $\left(U_{k} \backslash U_{k+1}, D_{k}\left(U_{k} \backslash U_{k+1}\right)\right.$ of the digraph $\left(U_{k}, D_{k}\right)$ restricted to $U_{k} \backslash U_{k+1}$. Define game $\left(U_{k+1}, v_{k+1}\right)$ by setting for every $U \subseteq U_{k+1}$,

$$
v_{k+1}(U)= \begin{cases}v_{k}(U) & \text { if } P_{D_{k}}\left(i_{k+1}\right) \cap U=\emptyset \\ v_{k}\left(U \cup\left(U_{k} \backslash U_{k+1}\right)\right)-\tau\left(U_{k+1}, r_{k}\right)\left|U_{k} \backslash U_{k+1}\right| & \text { else, }\end{cases}
$$

digraph $\left(U_{k+1}, D_{k+1}\right)$ given by

$$
(i, j) \in D_{k+1} \text { if }\left\{\begin{array}{l}
(i, j) \in D_{k} \text { or } \\
i \in P_{D_{k}}\left(i_{k+1}\right) \text { and } j \in S_{D_{k}}\left(U_{k} \backslash U_{k+1}\right) \cap U_{k+1} .
\end{array}\right.
$$

and let $r_{k+1}$ be the restricted game of $\left(U_{k+1}, v_{k+1}, D_{k+1}\right)$. Set $k=k+1$. Goto Step 2 .

Step 4 Assign $y_{1}=v(N)-\sum_{j \in N \backslash\{1\}} x_{j}$. Stop.

In every step of the algorithm, for $U_{k+1} \subset U_{k}$ satisfying condition (4.9) with respect to $\left(U_{k}, v_{k}, D_{k}\right)$, any player in $U_{k} \backslash U_{k+1}$ receives payoff $\tau\left(U_{k+1}, r_{k}\right)$. Observe that at any iteration the new found set $U_{k+1}$ is essential in $\left(U_{k+1}, r_{k+1}\right)$. If not, there exists an essential subset $S$ of $U_{k+1}$ with $r_{k+1}(S)=r_{k+1}\left(U_{k}\right)$, yielding payoff $y_{j}=0$ for all $j \in U_{k+1} \backslash S$. This contradicts that all players get positive payoff (because it is assumed that $N$ is essential). Since in any iteration the payoff of at least one player is determined, in at most $k=n-1$ iterations the algorithm stops with $U_{k+1}=\{1\}$ and player 1 getting what is left from $v(N)$ after all other players received their payoffs as determined by the algorithm. (Note that player 1 belongs to the player set of every game $\left(U_{k}, D_{k}\right)$ that appears in the algorithm.) In the remaining of this section we show that the algorithm indeed yields the nucleolus.

Let $K$ be such that $U_{K+1}=\{1\}$. To show that the algorithm is well-defined, it is needed that the results of Section 4 hold for every game $\left(U_{k}, r_{k}\right), k=1, \ldots, K$. This is shown in the next two lemmas. The first lemma states that for any $k=0,1, \ldots, K-1$ the digraph $\left(U_{k+1}, D_{k+1}\right)$ is acyclic and quasi-strongly connected with $i=1$ as its unique top-node.

Lemma 5.1 The digraph $\left(U_{k+1}, D_{k+1}\right)$ satisfies Assumption 2.5 for any $k=0,1, \ldots, K-1$.

Proof. Since $(N, D)$ satisfies Assumption 2.5, the statement is true for $k=0$. We now proceed by induction and suppose that the statement is true for $j=0, \ldots, k, k<K-1$. Then it remains to show that the statement is true for $j=k+1$. By the induction 
hypothesis we have that $\left(U_{k}, D_{k}\right)$ is acyclic and quasi-strongly connected and has $i=1$ as its unique top node. So, for any $j \neq 1$ in $U_{k+1}$ there is a directed path $i_{1}, \ldots, i_{m}$ in $\left(U_{k}, D_{k}\right)$ with $i_{1}=1$ and $i_{m}=j$. If any node $i_{k}, k=2, \ldots, m-1$ in this path is in $U_{k+1}$, then this path also exists in $\left(U_{k+1}, D_{k+1}\right)$. Otherwise, for any node $i_{h}$ on the path not in $U_{k+1}$, there exist two (not necessarily different) nodes $i_{r}, i_{s}$ on the path with $r \leq h \leq s$ such that $i_{r-1}, i_{s+1} \in U_{k+1}$ and $i_{r}, i_{s} \notin U_{k+1}$. Then by (5.11) we have that $\left(i_{r-1}, i_{s+1}\right) \in D_{k+1}$. Hence there is a directed path from $i=1$ to $i=j$ in $\left(U_{k+1}, D_{k+1}\right)$, showing $\left(U_{k+1}, D_{k+1}\right)$ is quasi-strongly connected with node 1 as top node. Because in $\left(U_{k+1}, D_{k+1}\right)$ there can only be a directed path from node $i$ to node $j$ if there is a directed path from $i$ to $j$ in $\left(U_{k}, D_{k}\right)$, the acyclicity of $\left(U_{k+1}, D_{k+1}\right)$ follows immediately from the fact that $\left(U_{k}, D_{k}\right)$ is acyclic.

The next lemma shows that any game $\left(U_{k}, v_{k}, D_{k}\right), k=0,1, \ldots, K$ satisfies the conditions of weak digraph monotonicity and weak digraph concavity. Again the proof is by induction, where Proposition 4.7 is used to show the weak digraph monotonicity.

Lemma 5.2 Let game with permission structure $(N, v, D)$ satisfy conditions (3.6) and (3.7). Then the game with permission structure $\left(U_{k}, v_{k}, D_{k}\right)$ satisfies these conditions on the player set $U_{k}$ for every $k=0, \ldots, K$.

Proof. We prove the proposition by induction on $k$. For $k=0$ both conditions (3.6) and (3.7) are satisfied by assumption. Proceeding by induction, assume that these conditions are satisfied for $j=0, \ldots, k, k<K-1$. By Lemma 5.1 the digraph $\left(U_{k}, D_{k}\right)$ satisfies Assumption 2.5. So, the game $\left(U_{k}, v_{k}, D_{k}\right)$ satisfies all conditions of Proposition 4.7.

To show that condition (3.6) holds for $\left(U_{k+1}, v_{k+1}, D_{k+1}\right)$, we have to show that $[U \subseteq$ $U_{k+1}$ and $U$ feasible in $\left.\left(U_{k+1}, D_{k+1}\right)\right] \Rightarrow v_{k+1}(U) \leq v_{k+1}\left(U_{k+1}\right)$. Since $P_{D_{k}}\left(i_{k+1}\right) \cap U_{k+1} \neq \emptyset$, we have that

$v_{k+1}\left(U_{k+1}\right)=v_{k}\left(U_{k+1} \cup\left(U_{k} \backslash U_{k+1}\right)\right)-\tau\left(U_{k+1}, r_{k}\right)\left|U_{k} \backslash U_{k+1}\right|=v_{k}\left(U_{k}\right)-\tau\left(U_{k+1}, r_{k}\right)\left|U_{k} \backslash U_{k+1}\right|$.

Next, let $U \subseteq U_{k+1}$ be a feasible subset of $U_{k+1}$ in $\left(U_{k+1}, D_{k+1}\right)$. We consider two cases, either $P_{D_{k}}\left(i_{k+1}\right) \cap U \neq \emptyset$ or $P_{D_{k}}\left(i_{k+1}\right) \cap U=\emptyset$. In the latter case we have that (i) $v_{k+1}(U)=v_{k}(U)$ and (ii) there is an arc between two nodes $i$ and $j$ of $U$ in the digraph $\left(U_{k+1}, D_{k+1}\right)$ if and only if there is also an arc between $i$ and $j$ in $\left(U_{k}, D_{k}\right)$. Hence $U$ is also feasible in $\left(U_{k}, D_{k}\right)$ and thus $v_{k+1}(U)=v_{k}(U)=r_{k}(U)$. Moreover, $\tau\left(U, r_{k}\right)=\frac{r_{k}\left(U_{k}\right)-r_{k}(U)}{\left|U_{k} \backslash U\right|+1} \geq$ $\tau\left(U_{k+1}, r_{k}\right)$ and thus $r_{k}\left(U_{k}\right)-r_{k}(U) \geq\left(\left|U_{k} \backslash U\right|+1\right) \tau\left(U_{k+1}, r_{k}\right)$. Hence

$$
\begin{aligned}
v_{k+1}(U) & =r_{k}(U) \leq r_{k}\left(U_{k}\right)-\left(\left|U_{k} \backslash U\right|+1\right) \tau\left(U_{k+1}, r_{k}\right) \\
& <v_{k}\left(U_{k}\right)-\left|U_{k} \backslash U_{k+1}\right| \tau\left(U_{k+1}, r_{k}\right)=v_{k+1}\left(U_{k+1}\right) .
\end{aligned}
$$


In case $P_{D_{k}}\left(i_{k+1}\right) \cap U \neq \emptyset$, we obtain from applying Proposition 4.7 to $\left(U_{k}, v_{k}, D_{k}\right)$, that $U \cup\left(U_{k} \backslash U_{k+1}\right)$ is feasible in $\left(U_{k}, D_{k}\right)$. From this it follows that

$$
\begin{aligned}
v_{k+1}(U) & =v_{k}\left(U \cup\left(U_{k} \backslash U_{k+1}\right)\right)-\tau\left(U_{k+1}, r_{k}\right)\left|U_{k} \backslash U_{k+1}\right| \\
& \leq v_{k}\left(U_{k}\right)-\tau\left(U_{k+1}, r_{k}\right)\left|U_{k} \backslash U_{k+1}\right|=v_{k+1}\left(U_{k+1}\right)
\end{aligned}
$$

because condition (3.6) holds for $\left(U_{k}, v_{k}, D_{k}\right)$.

Next we consider condition (3.7), i.e., we have to show that $\left[S \cup T=U_{k+1}\right.$ and $S, T$ feasible in $\left.\left(U_{k+1}, D_{k+1}\right)\right] \Rightarrow v_{k+1}(S)+v_{k+1}(T) \geq v_{k+1}(S \cap T)+v_{k+1}\left(U_{k+1}\right)$. Since $S \cup T=$ $U_{k+1}$ we have that $P_{D_{k}}\left(i_{k+1}\right) \cap S \neq \emptyset$ or $P_{D_{k}}\left(i_{k+1}\right) \cap T \neq \emptyset$ (or both). We first consider the case that both intersections are nonempty and thus also $P_{D_{k}}\left(i_{k+1}\right) \cap(S \cap T) \neq \emptyset$. Then $S^{\prime}=S \cup\left(U_{k} \backslash U_{k+1}\right), T^{\prime}=T \cup\left(U_{k} \backslash U_{k+1}\right)$ are feasible in $\left(U_{k}, D_{k}\right)$ and $S^{\prime} \cup T^{\prime}=U_{k}$, and thus it follows from condition (3.7) for $\left(U_{k}, v_{k}, D_{k}\right)$ that

$$
\begin{aligned}
v_{k+1}(S)+v_{k+1}(T) & =v_{k}\left(S^{\prime}\right)+v_{k}\left(T^{\prime}\right)-2 \tau\left(U_{k+1}, r_{k}\right)\left|U_{k} \backslash U_{k+1}\right| \\
& \geq v_{k}\left(S^{\prime} \cap T^{\prime}\right)+v_{k}\left(U_{k}\right)-2 \tau\left(U_{k+1}, r_{k}\right)\left|U_{k} \backslash U_{k+1}\right| \\
& =v_{k}\left((S \cap T) \cup\left(U_{k} \backslash U_{k+1}\right)\right)+v_{k}\left(U_{k}\right)-2 \tau\left(U_{k+1}, r_{k}\right)\left|U_{k} \backslash U_{k+1}\right| \\
& =v_{k+1}(S \cap T)+v_{k+1}\left(U_{k+1}\right),
\end{aligned}
$$

where the last equality follows from the fact that $v_{k+1}(S \cap T)=v_{k}\left((S \cap T) \cup\left(U_{k} \backslash U_{k+1}\right)\right)-$ $\tau\left(U_{k+1}, r_{k}\right)\left|U_{k} \backslash U_{k+1}\right|$ and $v_{k+1}\left(U_{k+1}\right)=v_{k}\left(U_{k}\right)-\tau\left(U_{k+1}, r_{k}\right)\left|U_{k} \backslash U_{k+1}\right|$. In case one of the sets $S$ and $T$ has a nonempty intersection with $P_{D_{k}}\left(i_{k+1}\right)$ and thus $P_{D_{k}}\left(i_{k+1}\right) \cap(S \cap T)=\emptyset$, suppose without loss of generality that $T \cap P_{D_{k}}\left(i_{k+1}\right)=\emptyset$. Then $S^{\prime}=S \cup\left(U_{k} \backslash U_{k+1}\right)$ and $T$ are feasible in $\left(U_{k}, D_{k}\right), S^{\prime} \cup T=N$ and thus it follows from condition (3.7) for $\left(U_{k}, v_{k}, D_{k}\right)$ that

$$
\begin{aligned}
v_{k+1}(S)+v_{k+1}(T) & =v_{k}\left(S^{\prime}\right)+v_{k}(T)-\tau\left(U_{k+1}, r_{k}\right)\left|U_{k} \backslash U_{k+1}\right| \\
& \geq v_{k}\left(\left(S^{\prime} \cap T\right)\right)+v_{k}\left(U_{k}\right)-\tau\left(U_{k+1}, r_{k}\right)\left|U_{k} \backslash U_{k+1}\right| \\
& =v_{k+1}(S \cap T)+v_{k+1}\left(U_{k+1}\right)
\end{aligned}
$$

where the last equality follows from the fact that $v_{k}\left(S^{\prime} \cap T\right)=v_{k}(S \cap T)=v_{k+1}(S \cap T)$ and $v_{k}\left(U_{k}\right)-\tau\left(U_{k+1}, r_{k}\right)\left|U_{k} \backslash U_{k+1}\right|=v_{k+1}\left(U_{k+1}\right)$.

In the remaining of this section we show that for $k=1, \ldots, K$, the game $\left(U_{k+1}, r_{k+1}\right)$ is the Davis-Maschler reduced game of the game $\left(U_{k}, r_{k}\right)$ with respect to the nucleolus. For a game $(N, v)$, let $T \subset N$ be a nonempty coalition and $y \in \mathbb{R}^{n}$ a payoff vector. Then the Davis-Maschler reduced game on $T$ at $y$ is the game $\left(T, v_{T}^{y}\right)$ given by $v_{T}^{y}(T)=$ $v(N)-x(N \backslash T)$ and $v_{T}^{y}(S)=\max _{Q \subseteq N \backslash T}(v(S \cup Q)-y(Q)), S \subset T, S \neq N$. Observe that in the definition of the reduced game only the values $y_{j}$ of the players $j \in N \backslash T$ appear. In 
the following, let $\left(U_{k+1}, r_{k}^{\prime}\right)$ denote the Davis-Maschler reduced game of the game $\left(U_{k}, r_{k}\right)$ on the set $U_{k+1}$ at $y$ with $y_{j}=\tau^{*}\left(r_{k}\right)=\tau\left(U_{k+1}, r_{k}\right)$ for $j \in U_{k} \backslash U_{k+1}$.

We first show the following lemma on the largest disjunctive feasible subset of a coalition $U$ in the digraph $\left(U_{k}, D_{k}\right)$. In the sequel we denote this set by $\sigma_{k}(U)$. Observe that for $U \subseteq N$ we have that $\sigma_{0}(U)=\sigma(U)$.

Lemma 5.3 For the game with permission structure $\left(U_{k}, v_{k}, D_{k}\right)$, let $U_{k+1} \subset U_{k}$ and $i_{k+1} \notin$ $U_{k+1}$ be the set and node as obtained in the iteration $k$ of the algorithm. Then for each $U \subseteq U_{k+1}$ we have that

$$
\begin{aligned}
& \text { 1. } \sigma_{k+1}(U)=\sigma_{k}(U) \text { if } S_{D_{k}}\left(\sigma_{k}(U)\right) \subset U_{k+1} \text {; } \\
& \text { 2. } \sigma_{k+1}(U)=\sigma_{k}\left(U \cup\left(U_{k} \backslash U_{k+1}\right)\right) \backslash\left(U_{k} \backslash U_{k+1}\right) \text { if } i_{k+1} \in S_{D_{k}}\left(\sigma_{k}(U)\right) \text {. }
\end{aligned}
$$

Proof. 1. Consider $U \subseteq U_{k+1}$ with $S_{D_{k}}\left(\sigma_{k}(U)\right) \subset U_{k+1}$. Clearly, then $\sigma_{k}(U)$ is feasible in $\left(U_{k+1}, D_{k+1}\right)$ and thus $\sigma_{k}(U) \subseteq \sigma_{k+1}(U)$. Next, suppose that there exists some player $i \in \sigma_{k+1}(U) \backslash \sigma_{k}(U)$. Then there is path $\left(a_{0}, a_{1}, \ldots, a_{l}\right)$ such that (i) $a_{0}=1$, (ii) $a_{l}=i$, (iii) $a_{t} \in U$ for all $t=1, \ldots, l-1$, and (iv) $\left(a_{t}, a_{t+1}\right) \in D_{k+1}$ for all $t=0, \ldots, l-1$. If $\left(a_{t}, a_{t+1}\right) \in D_{k}$ for all $t=0, \ldots, l-1$, then $i \in \sigma_{k}(U)$ and we get a contradiction with our assumption that $i \in \sigma_{k+1}(U) \backslash \sigma_{k}(U)$. So, there must exist a $t \in\{0, \ldots, l-1\}$ such that $\left(a_{t}, a_{t+1}\right) \notin D_{k}$. By definition of digraph $D_{k+1}$ it holds that $a_{t} \in P_{D_{k}}\left(i_{k+1}\right)$, which contradicts $S_{D_{k}}\left(\sigma_{k}(U)\right) \subset U_{k+1}$. Hence $\sigma_{k+1}(U)=\sigma_{k}(U)$.

2. Consider $U \subseteq U_{k+1}$ with $i_{k+1} \in S_{D_{k}}\left(\sigma_{k}(U)\right)$. If there is a player $i \in \sigma_{k+1}(U)$ then there is a path $\left(a_{0}, a_{1}, \ldots, a_{l}\right)$ such that (i) $a_{0}=1$, (ii) $a_{l}=i$, (iii), $a_{t} \in U$ for all $t=1, \ldots, l-1$, and (iv) $\left(a_{t}, a_{t+1}\right) \in D_{k+1}$ for all $t=0, \ldots, l-1$. We show that these four conditions also describe all elements of

$$
\sigma_{k}\left(U \cup\left(U_{k} \backslash U_{k+1}\right)\right) \backslash\left(U_{k} \backslash U_{k+1}\right)
$$

If $\left(a_{t}, a_{t+1}\right) \in D_{k}$ for all $t=0, \ldots, l-1$, then $i \in \sigma_{k}(U)$. Since $U \subseteq U_{k+1}$, it follows that $i \in \sigma_{k}\left(U \cup\left(U_{k} \backslash U_{k+1}\right)\right) \backslash\left(U_{k} \backslash U_{k+1}\right)$. Otherwise, if $\left(a_{t}, a_{t+1}\right) \in D_{k+1} \backslash D_{k}$ for some $t$, then $a_{t} \in P_{D_{k}}\left(i_{k+1}\right)$ and $a_{t+1} \in S_{D_{k}}\left(U_{k} \backslash U_{k+1}\right)$. So there is a path from $a_{t}$ to $a_{t+1}$ which contains only elements from $U_{k} \backslash U_{k+1}$. In the path $\left(a_{0}, a_{1}, \ldots, a_{l}\right)$, replace the arc $\left(a_{t}, a_{t+1}\right)$ by this path from $a_{t}$ to $a_{t+1}$.

Continuing in this way, we can change each arc in the path $\left(a_{0}, a_{1}, \ldots, a_{l}\right)$ that belongs to $D_{k+1} \backslash D_{k}$ by a path which consists only of elements from $U_{k} \backslash U_{k+1}$. So, we have a path from 1 to $i$ which consists only of elements from $U \cup\left(U_{k} \backslash U_{k+1}\right)$, implying that $i \in \sigma_{k}(U \cup$ $\left.\left(U_{k} \backslash U_{k+1}\right)\right)$. Since $i \notin U_{k} \backslash U_{k+1}$, we conclude that $i \in \sigma_{k}\left(U \cup\left(U_{k} \backslash U_{k+1}\right)\right) \backslash\left(U_{k} \backslash U_{k+1}\right)$. So, in both cases we have that $i \in \sigma_{k}\left(U \cup\left(U_{k} \backslash U_{k+1}\right)\right) \backslash\left(U_{k} \backslash U_{k+1}\right)$ and therefore we get

$$
\sigma_{k+1}(U)=\sigma_{k}\left(U \cup\left(U_{k} \backslash U_{k+1}\right)\right) \backslash\left(U_{k} \backslash U_{k+1}\right) .
$$


The next lemma shows that the game $\left(U_{k+1}, r_{k+1}\right)$ is the Davis-Maschler reduced game of the game $\left(U_{k}, r_{k}\right)$ with respect to the nucleolus.

Lemma 5.4 Let game with permission structure $(N, v, D)$ satisfy conditions (3.6) and (3.7). Then, for $k=0, \ldots, K$, the game $\left(U_{k+1}, r_{k+1}\right)$ is equal to the Davis-Maschler reduced game $\left(U_{k+1}, r_{k}^{\prime}\right)$ of the game $\left(U_{k}, r_{k}\right)$ on $U_{k+1}$ at $y$ with $y_{j}=\tau^{*}\left(r_{k}\right)$ for $j \in U_{k} \backslash U_{k+1}$.

Proof. For coalition $T \subseteq U_{k+1}$, we consider two cases, namely whether or not $S_{D_{k}}\left(\sigma_{k}(T)\right) \subset$ $U_{k+1}$. In case $S_{D_{k}}\left(\sigma_{k}(T)\right) \subset U_{k+1}$, Lemma 5.3.1 implies that $\sigma_{k+1}(T)=\sigma_{k}(T)$.

Further, since $P_{D_{k}}\left(i_{k+1}\right) \cap \sigma_{k}(T)=\emptyset$ we have by equation (5.10) in Step 3 of the algorithm that $v_{k+1}(T)=v_{k}(T)$ and thus $r_{k+1}(T)=r_{k}(T)$ because $\sigma_{k+1}(T)=\sigma_{k}(T)$. On the other hand, for the Davis-Mashler reduced game $\left(U_{k+1}, r_{k}^{\prime}\right)$ it holds for any $T \subset U_{k+1}$ that

$$
r_{k}^{\prime}(T)=\max _{Q \subseteq U_{k} \backslash U_{k+1}}\left(r_{k}(T \cup Q)-y(Q)\right)=r_{k}(T),
$$

because for any $Q \subseteq U_{k} \backslash U_{k+1}$ we have that

$$
r_{k}\left(T \cup Q^{\prime}\right)=v_{k}\left(\sigma_{k}(T \cup Q)\right)=v_{k}\left(\sigma_{k}(T)\right)=r_{k}(T),
$$

where the second equality follows since for any pair $j \in\left(T \backslash \sigma_{k}(T) \cup Q\right)$ and $i \in \sigma_{k}(T)$, it holds that $(i, j) \notin D_{k}$ and thus $\sigma_{k}(T \cup Q)=\sigma_{k}(T)$. Hence $r_{k}^{\prime}(T)=r_{k}(T)=r_{k+1}(T)$.

In case $S_{D_{k}}\left(\sigma_{k}(T)\right)$ is not a subset of $U_{k+1}$ we have that $P_{D_{k}}\left(i_{k+1}\right) \cap \sigma_{k}(T) \neq \emptyset$, because $i_{k+1}$ is the unique successor of $U_{k+1}$ in $U_{k} \backslash U_{k+1}$. So, by equation (5.10) in Step 3 of the algorithm we have that

$$
r_{k+1}(T)=v_{k+1}\left(\sigma_{k+1}(T)\right)=v_{k}\left(\sigma_{k+1}(T) \cup\left(U_{k} \backslash U_{k+1}\right)\right)-\tau\left(U_{k+1}, r_{k}\right)\left|U_{k} \backslash U_{k+1}\right| .
$$

From Lemma 5.3 we have that $\sigma_{k+1}(T) \cup\left(U_{k} \backslash U_{k+1}\right)=\sigma_{k}\left(T \cup\left(U_{k} \backslash U_{k+1}\right)\right)$ and so

$$
\begin{gathered}
r_{k+1}(T)=v_{k}\left(\sigma_{k}\left(T \cup\left(U_{k} \backslash U_{k+1}\right)\right)\right)-\tau\left(U_{k+1}, r_{k}\right)\left|U_{k} \backslash U_{k+1}\right|= \\
r_{k}\left(T \cup\left(U_{k} \backslash U_{k+1}\right)\right)-\tau\left(U_{k+1}, r_{k}\right)\left|U_{k} \backslash U_{k+1}\right| .
\end{gathered}
$$

To show that $r_{k+1}(T)=r_{k}^{\prime}(T)$ it remains to prove that the right-hand term in the equation

$$
r_{k}^{\prime}(T)=\max _{Q \subseteq U_{k} \backslash U_{k+1}}\left(r_{k}(T \cup Q)-\tau\left(U_{k+1}, r_{k}\right)|Q|\right)
$$

obtains its maximum for $U_{k} \backslash U_{k+1}$. To do so, denote $\bar{Q}=U_{k} \backslash U_{k+1}, V=T \cup \bar{Q}$ and, for $Q \subseteq \bar{Q}$, denote $W=U_{k+1} \cup Q$. Then (because of Lemma 5.3) the sets $\sigma_{k}(V)=\sigma_{k+1}(T) \cup \bar{Q}$ and $\sigma_{k}(W)=\sigma_{k}\left(U_{k+1} \cup Q\right) \supseteq U_{k+1}$ are feasible and satisfy $\sigma_{k}(V) \cup \sigma_{k}(W)=U_{k}$. By Lemma 
5.2 the game with permission structure $\left(U_{k}, v_{k}, D_{k}\right)$ satisfies weak digraph concavity and thus

$$
\begin{gathered}
r_{k}(V)+r(W)=v_{k}\left(\sigma_{k}(V)\right)+v_{k}\left(\sigma_{k}(W)\right) \geq v_{k}\left(U_{k}\right)+v_{k}\left(\sigma_{k}(V) \cap \sigma_{k}(W)\right)= \\
v_{k}\left(U_{k}\right)+v_{k}\left(\sigma_{k}(V \cap W)\right)=r_{k}\left(U_{k}\right)+r_{k}(V \cap W),
\end{gathered}
$$

where the second equality follows from the fact that $\sigma_{k}(V \cap W)=\sigma_{k}(V) \cap \sigma_{k}(W)$ because of the graph structure. With $V \cap W=(T \cup \bar{Q}) \cap\left(U_{k+1} \cup Q\right)=T \cup Q$ this yields

$$
\begin{aligned}
& r_{k}(T \cup \bar{Q})-r_{k}(T \cup Q) \geq r_{k}\left(U_{k}\right)-r_{k}\left(U_{k+1} \cup Q\right)> \\
& \quad \frac{r_{k}\left(U_{k}\right)-r\left(U_{k+1} \cup Q\right)}{|\bar{Q}|-|Q|+1}(|\bar{Q}|-|Q|)= \\
& \\
& \tau\left(U_{k+1} \cup Q\right)(|\bar{Q}|-|Q|) \geq \tau\left(U_{k+1}, r_{k}\right)(|\bar{Q}|-|Q|)
\end{aligned}
$$

by definition of $U_{k+1}$. Hence

$$
r_{k}(T \cup \bar{Q})-\tau\left(U_{k+1}, r_{k}\right)|\bar{Q}|>r_{k}(T \cup Q)-\tau\left(U_{k+1}, r_{k}\right)|Q|,
$$

for all $Q \subseteq \bar{Q}$, which shows that indeed

$$
r_{k}(T \cup \bar{Q})-\tau\left(U_{k+1}, r_{k}\right)|\bar{Q}|=\max _{Q \subseteq U_{k} \backslash U_{k+1}}\left(r_{k}(T \cup Q)-\tau\left(U_{k+1}, r_{k}\right)|Q|\right) .
$$

We now have the following proposition.

Proposition 5.5 Given game with permission structure $(N, v, D)$ satisfying the conditions (3.6) and (3.7), the algorithm described by the Steps 1-4 above yields the nucleolus of $(N, r)$.

Proof. In iteration $k=0$ the algorithm assigns in Step 2 the value $\tau^{*}\left(r_{0}\right)=\tau\left(U_{1}, r_{0}\right)=$ $\tau\left(U_{1}, r\right)$ to any player $j \in U_{0} \backslash U_{1}=N \backslash U_{1}$. According to Lemma 4.5, $\tau^{*}\left(r_{0}\right)$ is the nucleolus value of the players in $N \backslash U_{1}$. Applying Lemma 5.4 for $k=0$, the game $\left(U_{1}, r_{1}\right)$ is the DavisMaschler reduced game of the game $(N, r)$ with respect to the nucleolus values $y_{j}=\tau^{*}\left(r_{0}\right)$ of the players not in $U_{1}$. Since the nucleolus satisfies the Davis-Maschler reduced game consistency property, the nucleolus values of the reduced game $\left(U_{1}, r_{1}\right)$ are equal to the nucleolus values of the players of $U_{1}$ in the game $(N, r)$. In iteration $k=1$ the algorithm assigns in Step 2 the value $\tau^{*}\left(r_{1}\right)$ to any player $j \in U_{1} \backslash U_{2}$. According to Lemma 4.5, $\tau^{*}\left(r_{1}\right)$ is the nucleolus value of the players in $U_{1} \backslash U_{2}$ in the game $\left(U_{1}, r_{1}\right)$, and hence it is also the nucleolus value of these players in the game $(N, r)$. Continuing this reasoning we have that in any iteration $k$, the algorithm assigns in Step 2 the value $\tau^{*}\left(r_{k}\right)$ to any player $j \in U_{k} \backslash U_{k+1}$, which is the nucleolus value of the players in $U_{k} \backslash U_{k+1}$ in the game $(N, r)$. At the final iteration $K$ we have that $U_{K+1}=\{1\}$ and player 1 gets its nucleolus value in Step 4 of the algorithm. 


\section{Complexity of the algorithm}

For arbitrary veto-rich games the algorithm of Arin and Feltkamp (1997) to compute the nucleolus is an exponential time algorithm of the order $\mathcal{O}\left(n .2^{n-1}\right)$. Branzei et al. (2005) argue that applying the algorithm to the specific case of a peer group game the complexity reduces to a polynomial time algorithm of order $\mathcal{O}\left(n^{3}\right)$. They show that the algorithm given in their paper to find the nucleolus of a peer group game is a polynomial time algorithm of order $\mathcal{O}\left(n^{2}\right)$. In this section we show that the algorithm given in the previous section to find the nucleolus of the more general restricted game of a game with disjunctive permission structure is a polynomial time algorithm of order $\mathcal{O}\left(n^{4}\right)$. We first define the concept of a good set in a digraph.

Definition 6.1 For a digraph $(N, D)$ with $D \in \mathcal{D}^{N}$, a set $T \subset N$ is a good set, when (i) there is a unique top node in the subgraph $(T, D(T))$ of $(N, D)$ and for any other node $i$ in $T$ there is a path from this unique top node $i$ that only contains nodes in $T$,

(ii) the set $N \backslash T$ is connected, and

(iii) only the top node in $(T, D(T))$ has predecessors in $N \backslash T$.

We now have the following lemma.

Lemma 6.2 In any iteration $k$ of the algorithm, the set $U_{k} \backslash U_{k+1}$ is a good set.

Proof. Applying Corollary 4.8 to $\left(U_{k}, D_{k}\right)$ we have that the subgraph of $\left(U_{k}, D_{k}\right)$ restricted to $U_{k} \backslash U_{k+1}$ is a connected, acyclic directed graph with one top node, so condition (i) holds. Next, denote $T_{k}=U_{k} \backslash U_{k+1}$. Then $U_{k} \backslash T_{k}=U_{k+1}$. Therefore condition (ii) holds, because $U_{k+1}$ is feasible in $\left(U_{k}, D_{k}\right)$ and thus connected in $\left(U_{k}, D_{k}\right)$. Further, by applying the second statement of Proposition 4.7 to $\left(U_{k}, D_{k}\right)$ we have that $U_{k+1}$ has only one successor in $T_{k}=U_{k} \backslash U_{k+1}$. Let this only successor be node $j$ in $T_{k}$. Since the digraph $\left(U_{k}, D_{k}\right)$ is acyclic and quasi-strongly connected, there is a path from top node 1 in $\left(U_{k}, D_{k}\right)$ to any other node in $U_{k}$, so also to any node in $T_{k}$. Since $j$ is the only successor of $U_{k+1}$ in $T_{k}$, any path from 1 to some node $h \in T_{k}$ must contain the node $j$. Moreover, the path from $j$ to $h$ can not contain nodes not in $T_{k}$, otherwise $U_{k+1}$ has more than one successor in $T_{k}$. Hence $j$ is also a top node in $T_{k}$ such that for any other node in $T_{k}$ there is a path from $j$ to this node that only contains nodes in $T_{k}$.

Lemma 6.2 implies that in Step 2 of the algorithm the set $U_{k+1}$ that we must find is such that its complement $U_{k} \backslash U_{k+1}$ is a good set. Conversely, when $\mathcal{T}_{k}$ is the collection of all good sets in $\left(U_{k}, D_{k}\right)$, then the search for $U_{k+1}$ can be restricted to sets in the collection $U_{k} \backslash T_{k}, T_{k} \in \mathcal{T}_{k}$. The next lemma says that in a game with permission structure $(N, v, D)$ there is precisely one good set for any player $j \in N$. Applying this to $\left(U_{k}, D_{k}\right)$ this means 
that at iteration $k$ of the algorithm the number of good sets is equal to $\left|U_{k}\right|$. Observe that $j$ itself is a singleton good set if $j$ has no successors.

Lemma 6.3 Let $(N, D)$ be a digraph with $D \in \mathcal{D}^{N}$. Then for any node $j \in N$ there is exactly one good set $T$ such that $j$ is the unique top node in $T$.

Proof. Recall from Section 2.3 that the set $\bar{S}_{D}(j)$ of all complete subordinates of $j$ is the set of nodes $i$ such that any path from top node 1 in $(N, D)$ to node $i$ contains node $j$. It is straightforward to verify that $\bar{S}_{D}(j)$ is a good set having node $j$ as its unique top node. Next, suppose that there are two good sets with $j$ as their unique top node, say $T_{1}$ and $T_{2}$ and, w.l.o.g., suppose that $T_{1} \backslash T_{2}$ is non-empty. Consider some node $h \in T_{1} \backslash T_{2}$. By definition of a good set we know that any path from top player 1 to the player $h$ contains the node $j$. However, $N \backslash T_{2}$ does not contain $j$ and so there is no path from top node 1 to $h$ in $N \backslash T_{2}$, contradicting condition (ii) of Definition 6.1.

We are now ready to consider the complexity of the algorithm.

Proposition 6.4 The complexity of the algorithm is of order $\mathcal{O}\left(n^{4}\right)$.

Proof. First, in iteration $k$ we have to find all good sets in $U_{k}$. To find the good set with some player $j$ in $U_{k}$ as its unique top node, delete player $j$ from $U_{k}$. Then the good set consists of player $j$ and all nodes in $U_{k}$ that are no longer connected to player 1 when player $j$ is deleted. Since $U_{k}$ contains at most $n-1$ nodes not equal to 1 , this requires at most $\mathcal{O}\left(n^{2}\right)$ actions to find the good set of node $j$. So, it requires at most $\mathcal{O}\left(n^{3}\right)$ actions to find all $n-1$ good sets of all players $j \neq 1$. Next, at each iteration $k$ we need to calculate the number $\tau\left(U_{k} \backslash T, r_{k}\right)$ for any good set $T$. For this we need at most $O(n-1) m_{k}$ actions, where $m_{k}$ is the number of actions to find all values $v_{k}(U), U \subseteq U_{k}$ in Step 3 of iteration $k-1$. Clearly $m_{0}=1$. Further, from equation (5.10) in Step 3 of the algorithm it follows that we need $m_{k-1}$ actions to find $v_{k}(U)$ if $P_{D_{k}}\left(i_{k+1}\right) \cap U=\emptyset$. Otherwise $m_{k-1}$ actions are needed to calculate $v_{k}(U)=v_{k-1}\left(U \cup\left(U_{k-1} \backslash U_{k}\right)\right)$ and $\mathcal{O}(1)$ actions are needed for calculating $\tau\left(U_{k}, r_{k-1}\right)\left|U_{k-1} \backslash U_{k}\right|$ and for substraction, because $\tau\left(U_{k}, r_{k-1}\right)$ was already found before. Hence $m_{k}=m_{k-1}+\mathcal{O}(1)$. Together with $m_{0}=1$ this yields that $m_{k} \leq \mathcal{O}(n)$. Since the number of iterations is at most equal to $n$, it follows that the complexity of the algorithm is given by $n \cdot\left(O\left(n^{3}\right)+O\left((n-1) m_{k}\right)\right)=O\left(n^{4}\right)$.

\section{References}

Arin, J., and Feltkamp, V. (1997), The nucleolus and kernel of veto-rich transferable utility games, International Journal of Game Theory 26, 61-73. 
Bondareva, O. (1962), The theory of the Core in an $n$-person game, Vestnik Leningrad. Univ. 13, 141-142 (in Russian).

Brânzei, R., Fragnelli, V., and Tijs, S. (2002), Tree connected line graph peer group situations and line graph peer group games, Mathematical Methods of Operations Research 55, 93-106.

Brânzei, R., Solymosi, T., and Tijs, S. (2005), Strongly essential coalitions and the nucleolus of peer group games, International Journal of Game Theory 33, 447-460.

Brink, R. van den (1997), An axiomatization of the disjunctive permission value for games with a permission structure, International Journal of Game Theory 26, 27-43.

Brink, R. van den, and Gilles, R. P. (1996), Axiomatizations of the conjunctive permission value for games with permission structures, Games and Economic Behavior 12, 113126.

Brune, S. (1983), On the regions of linearity for the nucleolus and their computation, International Journal of Game Theory 12, 47-80.

Gilles, R. P., and Owen, G. (1994), Cooperative games and disjunctive permission structures, Department of Economics, Virginia Polytechnic Institute and State University, Blacksburg, Virginia.

Gilles, R. P., Owen, G., and Brink, R. van den (1992), Games with permission structures: the conjunctive approach, International Journal of Game Theory 20, 277-293.

Gillies, D.B. (1953), Some Theorems on n-Person Games, Princeton University Press, Princeton, NJ.

Huberman, G. (1980), The nucleolus and essential coalitions, in: A. Bensoussan and J. Lions (eds.) Analysis and Optimization of Systems, Lecture Notes in Control and Information Sciences 28, Springer, Berlin, pp. 416-422.

Kohlberg, E., (1979), On the nucleolus of a characteristic function game, SIAM Journal of Applied Mathematics 20, 62-66.

Muto, S., Potters, J., and Tijs, S. (1989), Information market games, International Journal of Game Theory 18, 209-226.

Myerson, R. B. (1977), Graphs and cooperation in games, Mathematics of Operations Research 2, 225-229. 
Reynierse, J., and Potters, J. (1998), The $\mathcal{B}$-nucleolus of TU-games, Games and Economic Behavior 24, 77-96.

Schmeidler, D. (1969), The nucleolus of a characteristic function game, SIAM Journal on Applied Mathematics 17, 1163-1170.

Shapley, L. S. (1953), A value for N-person games, in: Contributions to the Theory of Games, Vol II (eds. H. W. Kuhn, and A. W. Tucker), Princeton University Press, Princeton, 307-317.

Shapley, L.S. (1967), On balanced sets and cores, Naval Research Logistics Quarterly 14, 453-460. 